\title{
Highly efficient hybrid fiber taper coupled microsphere laser
}

\author{
Ming Cai and Kerry Vahala \\ Department of Applied Physics, California Institute of Technology, Pasadena, California 91125
}

Received January 17, 2001

\begin{abstract}
A novel hybrid fiber taper is proposed and demonstrated as the coupler in a microsphere laser system. The pump wave and the laser emission, respectively, are more efficiently coupled to and from the sphere modes with this taper structure. A 980-nm pumped erbium-ytterbium codoped phosphate microsphere laser is demonstrated in the 1550-nm band. As much as $112 \mu \mathrm{W}$ of single-frequency laser output power was measured, with a differential quantum efficiency of $12 \%$. (C) 2001 Optical Society of America

OCIS codes: $140.4780,140.3510,230.3990,230.1150$.
\end{abstract}

High- $Q$ dielectric microcavities, in which light is trapped internally as whispering-gallery modes (WGMs), have attracted interest in applications as diverse as quantum optics and optical communications. ${ }^{1-4}$ Numerous coupling devices, such as high-index prisms under total internal reflection, ${ }^{1}$ half-block fiber couplers, ${ }^{5}$ angle-polished fiber tips, ${ }^{6}$ and tapered optical fibers, ${ }^{2,7}$ have been developed by several research groups. Among these, the tapered fibers have been shown to provide the most robust and efficient coupling to the WGMs without degrading the qualities of the microspheres. ${ }^{8}$

As one application of the taper coupling technique, we recently demonstrated an erbium-ytterbium codoped phosphate microsphere laser in the $1550-\mathrm{nm}$ band. ${ }^{4}$ A single 1550-nm fiber was tapered to serve dual purposes of coupling the optical pump wave at $980 \mathrm{~nm}$ into the microsphere and collecting the resultant laser emission.

In this Letter we propose a novel hybrid fiber taper-based laser coupling configuration in which the microsphere is coupled with a continuous fiber taper that is a combination of $980-\mathrm{nm}$ single-mode fiber (SMF) and 1550-nm SMF. The pump source and the laser emission are then both guided in a SMF to or from the taper region. This structure facilitates excellent power transfer efficiency between the microresonator and the respective pump-input and emission-output nontapered fiber regions. In addition, the measured laser output power of $112 \mu \mathrm{W}$ is more than an order of magnitude larger than the previous result reported in Ref. 4. The differential quantum efficiency is $12 \%$.

As described in Ref. 4, a 1550-nm band microsphere laser is formed by contact of a tapered fiber with an erbium-ytterbium codoped glass microsphere. The 980-nm pump source is guided from one end of the fiber to the taper-microsphere coupling region, where the taper has a waist of $1.5-2 \mu \mathrm{m}$. In the taper region, the SMF mode is converted into air-cladding taper modes that extend into the air surrounding the taper. Proper taper design ensures that coupling is primarily to the fundamental taper mode. Power transfer to the microsphere occurs over the region in which the taper is in proximity to the sphere WGMs. Similarly, the 1550-nm band laser emission is coupled back into the taper and is ultimately converted into a SMF mode.
To pump the microsphere efficiently, one must obtain good phase matching between the fundamental taper mode and a WGM of the sphere. Previously, ${ }^{4}$ the fiber taper was formed with a single standard 1550-nm SMF. The pump source and the laser emission were both guided in the single tapered fiber. The 1550-nm SMF generally has a cutoff wavelength at $1250 \mathrm{~nm}$. As a result, for the $980-\mathrm{nm}$ pump source it is actually a multimode waveguide and both $\mathrm{LP}_{01}$ (the fundamental fiber mode) and $\mathrm{LP}_{11}$ (the first higher-order fiber mode) pump modes can exist with comparable powers. To keep the taper loss low, one usually prepares the taper such that the slowness criterion ${ }^{9}$ is satisfied. Under this condition, $\mathrm{LP}_{01}$ will most likely transit into the fundamental taper mode $\mathrm{HE}_{11}$ while $\mathrm{LP}_{11}$ transits into the higher-order taper modes $\mathrm{HE}_{21}, \mathrm{TM}_{01}$, and $\mathrm{TE}_{01}$ of the taper region. Hence the higher-order taper modes $\mathrm{HE}_{21}, \mathrm{TM}_{01}$, and $\mathrm{TE}_{01}$ can carry a large fraction of pump power. Once it is phase matched to the fundamental taper mode $\mathrm{HE}_{11}$, the sphere fundamental mode will mismatch to higher-order taper modes $\mathrm{HE}_{21}, \mathrm{TM}_{01}$, and $\mathrm{TE}_{01}$. Replacing the $1550-\mathrm{nm} \mathrm{SMF}$ by a $980-\mathrm{nm}$ $\mathrm{SMF}$ is a possible alternative for fabrication of the coupling taper; however, 1550-nm lasing output coupling is empirically found to be difficult in this case, exhibiting excessive loss.

To avoid this problem, we propose a hybrid fiber taper coupling configuration, which includes both a 980-nm and a 1550-nm SMF, as shown in Fig. 1. The use of such a hybrid taper coupler guarantees that the pump wave is properly launched from the fundamental fiber mode $\mathrm{LP}_{01}$. The laser emission coupling to the 1550-nm SMF remains unchanged and also highly efficient.

The hybrid fiber taper was prepared in two steps. First, a section of 1550-nm SMF was spliced with a section of 980-nm SMF. It was then put on the top of a hydrogen flame, with the splice in the center of the heating source. We tapered the fiber by stretching it slowly apart by $\sim 2.55 \mathrm{~cm}$ in total to obtain a narrow waist of approximately 1-2 $\mu \mathrm{m}$ and a relatively low taper loss. Before tapering, the hybrid fiber had a loss of less than $0.2 \mathrm{~dB}$, which resulted from splice and connector losses. An additional 0.5-dB loss was measured after tapering.

Inasmuch as the taper modes are defined by the taper size and the material's refractive index (i.e., the old core is vestigial), the splice in the center region of the 


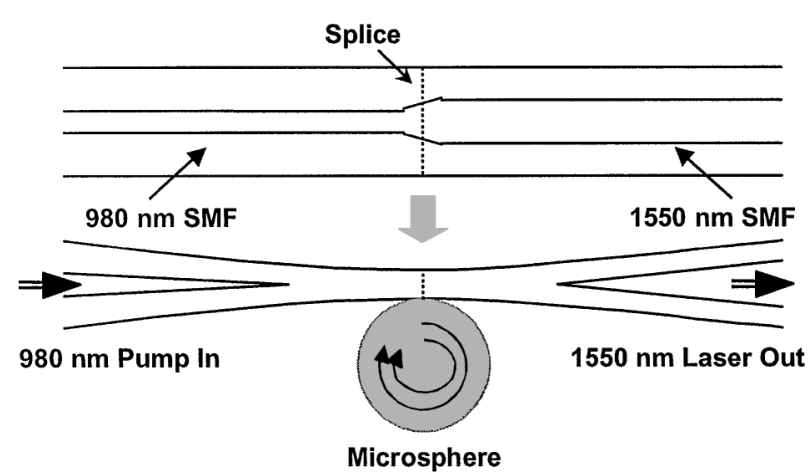

Fig. 1. Schematic of hybrid fiber taper coupling to a microsphere laser.

hybrid taper has no deleterious effect on the performance of this taper compared with that of normal (nonhybrid) tapers. However, as illustrated below, the hybrid fiber taper has the significant advantage that it transitions to SMF fiber optimized for propagating either the $980-\mathrm{nm}$ pump source or the $1550-\mathrm{nm}$ laser emission.

The diameter of the erbium-ytterbium codoped phosphate microsphere in this experiment was $\sim 45 \mu \mathrm{m}$, and the taper size that best matched the sphere was $\sim 1.5 \mu \mathrm{m}$. The characteristics of the phosphate glass and the fabrication of the microsphere are the same as described in Ref. 4.

Figure 2 shows a magnified image of the taper-sphere coupling system. The hybrid taper was placed underneath the sphere with the 980-nm SMF at the left side and the 1550-nm SMF at the right side. The pump wave was launched from a $980-\mathrm{nm}$ single-mode tunable external-cavity laser. The bright (green) ring encircling the equator of the sphere is due to spontaneous emission from the upconversion process. It traces the path taken by the $980-\mathrm{nm}$ pump wave within the sphere. In this case, it shows that the pump wave is resonantly coupled into a fundamental WGM, producing a narrow equatorial-ring gain region. The fiber stem is for convenience in handling and is not necessarily perpendicular to the excited equatorial plane.

Lasing action was observed with the hybrid fiber taper coupled microsphere by tuning of the pump wavelength to a pump band resonance of the sphere. The pump polarization state and the taper-sphere contact position were also adjusted carefully to maximize the pump coupling to the sphere. Figure 3 shows a typical laser spectrum (as collected from the $1550-\mathrm{nm}$ SMF end) read from an optical spectrum analyzer with resolution of $0.08 \mathrm{~nm}$. To resolve the fine spectral features of the laser, we used a highfinesse ( 5000) scanning Fabry-Perot etalon with a spectral resolution of a few megahertz to analyze the spectrum. Figure 4 shows the resultant microsphere laser spectrum. For reference, a single-frequency laser with known linewidth of $\sim 300 \mathrm{kHz}$ is also shown. The spectral output shows the single-frequency nature of the microsphere laser.

A plot of laser power collected from the SMF as a function of total pump power absorbed and scattered is shown in Fig. 5. As much as $112 \mu \mathrm{W}$ of output laser power was measured with the laser still remaining single mode. The differential quantum efficiency of this laser was calculated to be $\sim 12 \%$. In our previous

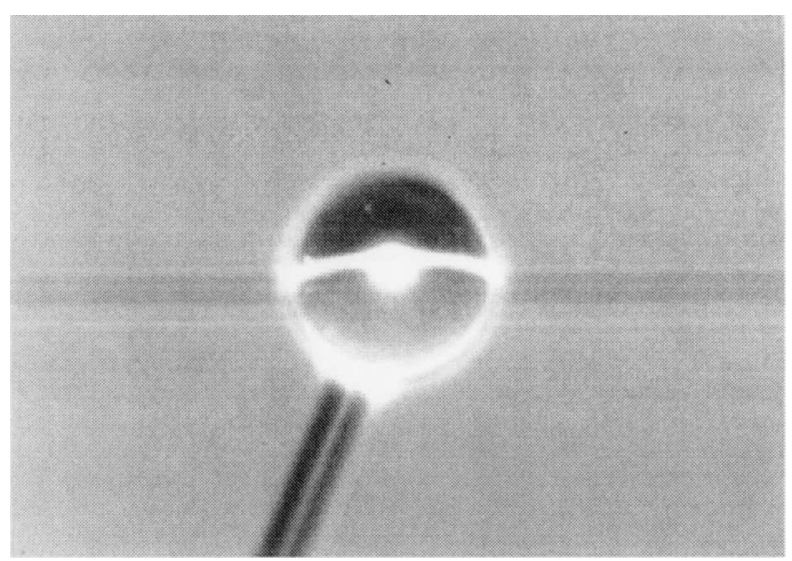

Fig. 2. Image of a hybrid fiber taper-pumped microsphere system.

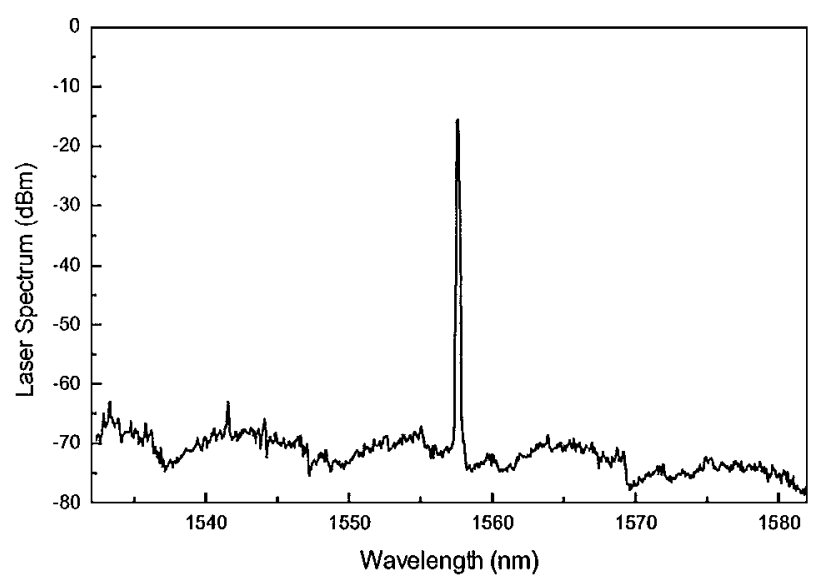

Fig. 3. Laser spectrum in the $1550-\mathrm{nm}$ band measured from the 1550-nm fiber end.

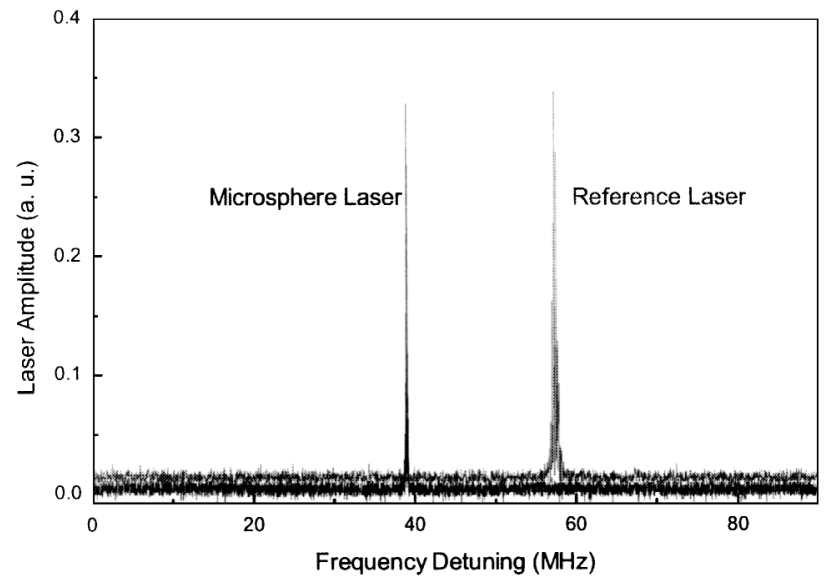

Fig. 4. Laser spectrum observed with a Fabry-Perot etalon, showing the single-mode nature of the microsphere laser. The reference laser scan is from a single-mode laser with a linewidth of $300 \mathrm{kHz}$. 


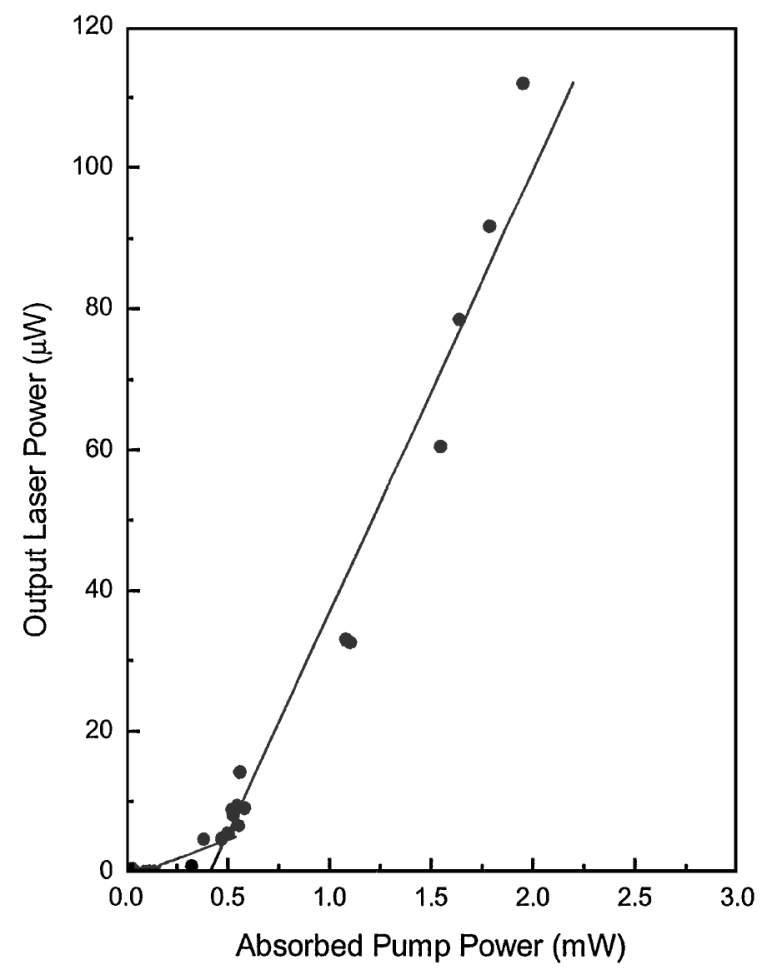

Fig. 5. Measured total output laser power versus absorbed pump power in the microsphere.

study $^{4}$ in which a conventional 1550-nm SMF taper was used, the quantum efficiency was $\sim 2.6 \%$; it was $\sim 0.037 \%$ in the research reported in Ref. 3 , in which the prism coupling technique was used.
In summary, a hybrid fiber taper has been proposed and demonstrated as the coupler in a fiber-microsphere laser system. Both the pump source and the laser emission were guided to and from the taper region in fundamental modes, thereby permitting more-efficient power coupling. The measured output laser power was greatly improved to $112 \mu \mathrm{W}$, with no sign of saturation, and the differential quantum efficiency was as high as $12 \%$.

This research was supported by the Defense Advanced Research Projects Agency, the U.S. Office of Naval Research, and the Caltech Lee Center. M. Cai's e-mail address is mingcai@caltech.edu.

\section{References}

1. M. L. Gorodetsky, A. A. Savchenkov, and V. S. Ilchenko, Opt. Lett. 21, 453 (1996).

2. M. Cai, G. Hunziker, and K. Vahala, IEEE Photon. Technol. Lett. 11, 686 (1999).

3. V. Sandoghdar, F. Treussart, J. Hare, V. LefevreSeguin, J. M. Raimond, and S. Haroche, Phys. Rev. A 54, 1777 (1996).

4. M. Cai, O. Painter, K. J. Vahala, and P. C. Sercel, Opt. Lett. 25, 1430 (2000).

5. A. Serpenguzel, S. Arnold, and G. Griffel, Opt. Lett. 20, 654 (1995).

6. V. S. Ilchenko, X. S. Yao, and L. Maleki, Opt. Lett. 24, 723 (1999).

7. J. C. Knight, G. Cheung, F. Jacques, and T. A. Birks, Opt. Lett. 22, 1129 (1997).

8. M. Cai, O. Painter, and K. Vahala, Phys. Rev. Lett. 85, 74 (2000).

9. A. W. Synder and J. D. Love, Optical Waveguide Theory (Chapman \& Hall, London, 1983). 